\title{
Impact tensile cracking behavior of oxide scale in Q235 strip iron
}

\author{
Shang Wang ${ }^{1}$, Wenpei $\mathrm{Li}^{1}$, Xiaochen Wang ${ }^{1, *}$, Rui $\mathrm{Ai}^{1}$, Quan Yang ${ }^{1}$, and Zhongde Shan ${ }^{2}$ \\ ${ }^{1}$ Institute of Engineering Technology, University of Science and Technology Beijing, Beijing 100083, \\ China \\ ${ }^{2}$ State Key Laboratory of Advanced Forming Technology and Equipment, China Academy of \\ Machinery Science and Technology, Beijing 100083, China
}

\begin{abstract}
Scanning electron microscopy (SEM) is used to observe the fracture surface morphology of Q235 steel. The cracking law/crack rule principle of strip surface under tension is studied via universal tensile testing machine. The results show that the thickness of the scale is relatively uniform, about $10 \mu \mathrm{m}$, and the structure is dense and well attached to the matrix. Tensile experiments show that as the strain increases, the cracks increase slowly in the initial stage, rapidly in the middle period, and slowly in the final period. When the strain exceeds $0.15 \%$, the cracks are difficult to increase. In order to avoid direct erosion of the abrasive on the substrate, a reasonable range of tension setting should be $0.15 \%-0.18 \%$ in the pretreatment process without acid descaling.
\end{abstract}

\section{Introduction}

During the hot rolling and subsequent cooling on the strip, the surface forms a dense, brittle oxide scale (commonly known as scales). Before the cold rolling or the galvanizing process, the strip must be completely removed to ensure the surface quality of the finished product [1-4].

Before the traditional pickling line and the more researched acid-free descaling production line, there is a pull straightening unit. After the pull-bending combination of the tension leveler, a large number of cracks and partial exfoliation are treated on the scale surface, which provides good conditions for pickling and acid-free descaling [5-7].

According to those researches on scale cracking behavior under tension, Evans [8] believes that the evolution of internal defects in oxide layers under tension is the root cause of cracks, and based on fracture mechanics theory, the critical strain $\varepsilon_{\mathrm{c}}$ of scale cracking is proposed as:

$$
\varepsilon_{\mathrm{c}}=\frac{K_{\mathrm{IC}}}{E F(\pi c)^{1 / 2}} \text {. }
$$

Here, $K_{\mathrm{IC}}$ is the critical stress intensity factor, $c$ is half the defect length, $E$ is the Young's modulus, and $F$ is the value depending on the defect shape, size, and position.

\footnotetext{
*Corresponding author: wangxiaocheng@ustb.edu.cn
} 
Robertson and Manning [9] based on the theory of fracture mechanics consider the critical energy release of cracking to obtain the critical strain $\varepsilon_{\mathrm{c}}$ for scale cracking:

$$
\varepsilon_{\mathrm{c}}=\left(\frac{2 \gamma}{F^{2} \pi E c}\right)^{1 / 2} \text {. }
$$

Here, $\gamma$ is the fracture surface energy.

$\mathrm{Nagl}$ and Evans [10] analyzed the relationship between the cracking behavior and the thickness of scales under tension and found that the cracking stress/strain decreases with increasing thickness. It is believed that the thicker the oxide layer is, the greater the defect length is. The experimental results are consistent with the theoretical derivation of Evans [8] and Robertson [9]. Chaudhuri and Rolls [11] conducted a large number of experiments on the gradual cracking process of the oxide layer under tension, and fitted a parabolic relationship between crack width and stress/strain, and pointed out that the generation of new cracks requires greater stress/strain on the substrate.

Zhang et al. [12] and Bian et al. [13] found that tension can cause significant cracking of the scale layer, and the spalling effect is not obvious. He et al. [14] based on the research results of Le et al. [15] and combined with the experiment of rolling stop, proposed the cracking model of the scale layer under rolling action. It is believed that the pressure bite of the roller causes the scale layer to be under tension, when the stress exceeds the critical value. Wang and $\mathrm{Li}$ [6-7] analyzed the mechanism of tension and scale reduction in detail, and concluded that the extension rate should be $1 \%$, continue to increase tension, and the effect of removing scales is not obvious, but it has a number of negative effects on safe production.

These studies mainly focused on the first cracking of the oxide layer, but the regulation of the continuous cracking caused by the tension increase has not been paid much attention. The fracture criteria of Evans [8] and Robertson et al. [9] are based on the existence of internal faults in the scales, but with the increase of cracks, the regularity of cracking and cracking in the absence of defects is not explained in detail. Chaudhuri and Rolls [11] also through experimental observations, it is proposed that the generation of new cracks needs to continue to increase strain, however, the reason and mechanism are not demonstrated.Wang and $\mathrm{Li}$ [6-7] considered that the excessive expansion rate has no significant effect on improving the descaling effect, but did not improve theoretical analysis or experimental verification.

In order to improve the efficiency of acid-free descaling, the surface morphology of Q235 hot rolled strip was observed. The tensile cracking and evolutionary behaviors of the scales were analyzed by tensile tests to provide support for the parameter design of field tension.

\section{The Scale cross-section observation}

\subsection{The Cross section observation experiment method}

The experimental steel materials were taken from the Q235 hot-rolled strip of an iron and steel enterprise's actual production line with a width of $320 \mathrm{~mm}$ and a thickness of $3.5 \mathrm{~mm}$. The following samples were taken from the middle of the original steel strip. The chemical composition is shown in Table 1.

Table 1. Chemical composition of carbon steel \%.

\begin{tabular}{|c|c|c|c|c|}
\hline $\mathrm{C}$ & $\mathrm{Mn}$ & $\mathrm{Si}$ & $\mathrm{S}$ & $\mathrm{P}$ \\
\hline 0.216 & 1.250 & 0.183 & 0.041 & 0.0398 \\
\hline
\end{tabular}


A sample with a length and width of $10 \mathrm{~mm}$ was prepared by wire cutting. The surface of the sample was cleaned by an ultrasonic cleaning instrument, then washed with alcohol, dried and sectioned. The section was observed using a ZEISS ULTRA 55 scanning electron microscope.

\subsection{Experimental results}

Fig. 1 is a cross-sectional topography of a Q235 scale layer observed by a scanning electron microscope. From the cross-section morphology of the scale layer, it can be seen that the scale layer formed on the surface of the hot-rolled strip is continuous and dense, with a uniform thickness of about $10 \mu \mathrm{m}$, and no obvious delamination, indicating that there is an obvious $\mathrm{Fe}_{3} \mathrm{O}_{4} / \mathrm{Fe}$ eutectoid structure. $\mathrm{FeO}$ in the layer will occur $4 \mathrm{FeO}=\mathrm{Fe}_{3} \mathrm{O}_{4}+\mathrm{Fe}$ eutectoid transformation [16-17].

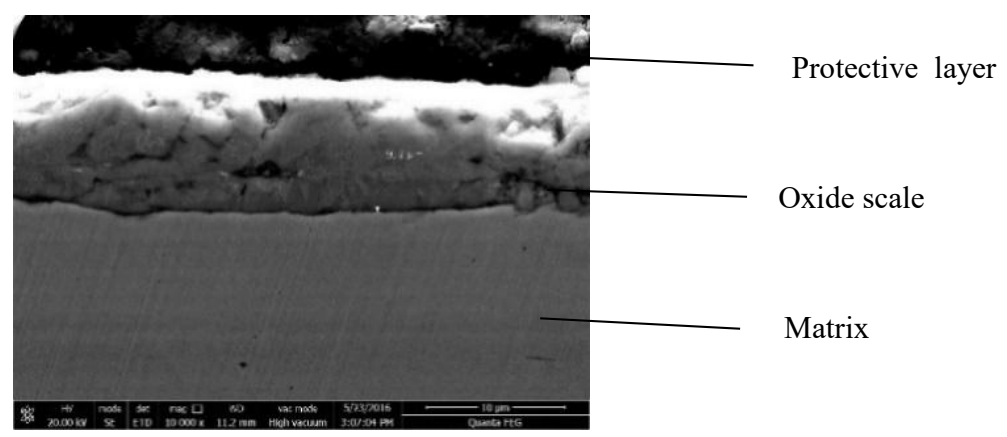

Fig. 1. Cross-section morphology of the oxide scale.

\section{Tension test}

\subsection{Tensile test method}

The tensile test specimen was taken by wire cutting. The length direction (tensile direction) of the specimen was the rolling direction. The specific shape and dimensions were as shown in Fig. 2 . The surface morphology of the tensile specimen was observed using a 4XB-TV metalloscope, and a specimen was selected without cracks to perform a tensile test. Apply specific strain to the sample using MTS Universal tensile testing machine. The displacement control is $0.01 \mathrm{~mm} \cdot \mathrm{min}-1$. Hold $1 \mathrm{~min}$ after reaching pre-set strain. Remove the sample and wipe the surface with an alcohol swab, using 4XB -TV type metalloscope to observe the surface morphology. In the experiment, the strain ranged from $0.04 \%$ to $0.20 \%$ and the step size was $0.01 \%$.

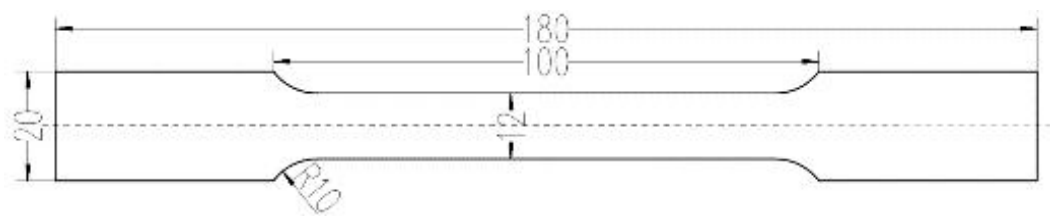

Fig. 2. Tensile specimen dimensions (unit: $\mathrm{mm}$ ).

\subsection{Experimental Results and Analysis}


The surface cracks of each sample were observed by 4XB-TV metallographic microscope (200 magnifications), and the cracks was counted in the region of $500 \mu \mathrm{m}$ in width at the center of the pattern. Fig. 3 shows the relationship between the number of cracks and the strain of each sample in the experiment, and Fig. 4 shows the cracking microstructure of the oxide layer under different strains.

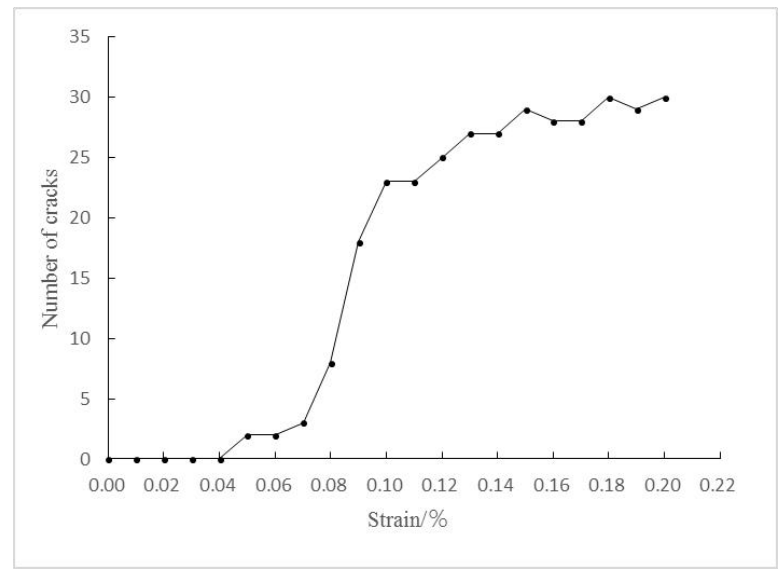

Fig. 3. Strain-crack curve of the oxide scale.

From Fig. 3 and Fig. 4, a strip of scaly oxide layer adheres to the surface of the strip, pits and pits are present on the surface; the cracks produced under tension are approximately linear and the direction is approximately perpendicular to the tension direction; as the tension increases, the increase of the scale cracks on the sample surface was slower, then fast, and later slower.

When the strain is $0.05 \%$, cracks appear (two at the same time) on the oxide layer of the sample surface, as shown in Fig. 4(a). As the strain continues to increase, the number of cracks begins to increase, the number of cracks increases slowly in the range of $0.05 \%$ to $0.07 \%$, and there is no regularity in the position, and the crack spacing varies in size, as shown in Figs. 4(a) and 4(b). When the strain reaches the range of $0.08 \%$ to $0.10 \%$, the number of cracks increases sharply with the increase of strain, and the crack direction is approximately perpendicular to the tensile direction and the parallelism is good, as shown in Fig. 4(c) and Fig. 4(d). When the strain exceeds $0.10 \%$, the number of cracks changes little with strain continues to increase, and the crack spacing tends to be uniform, as shown in Fig. 4(d) and Fig. 4(e). When the strain exceeds $0.15 \%$, the number of cracks in the sample almost no longer increases and reaches saturation state, as shown in Fig. 4(e) and Fig. 4(f).

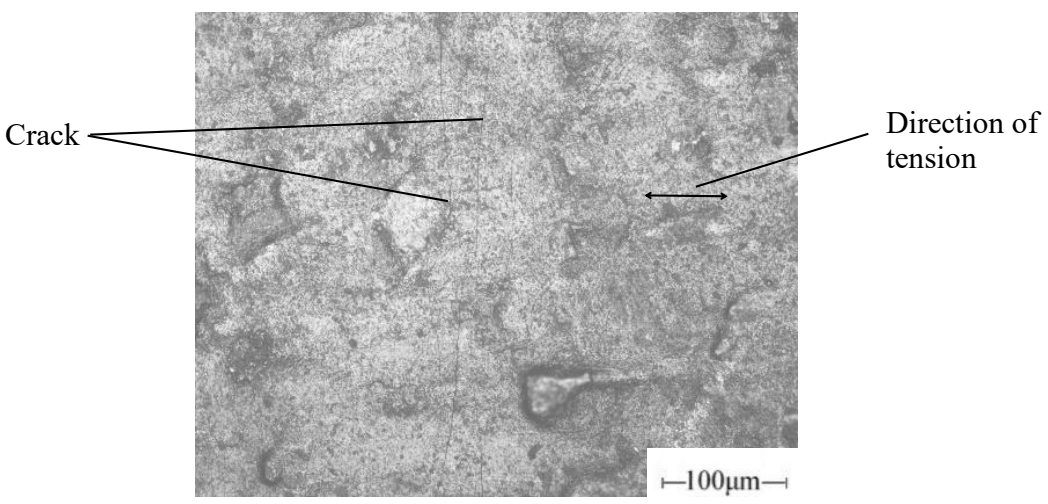

(a) 


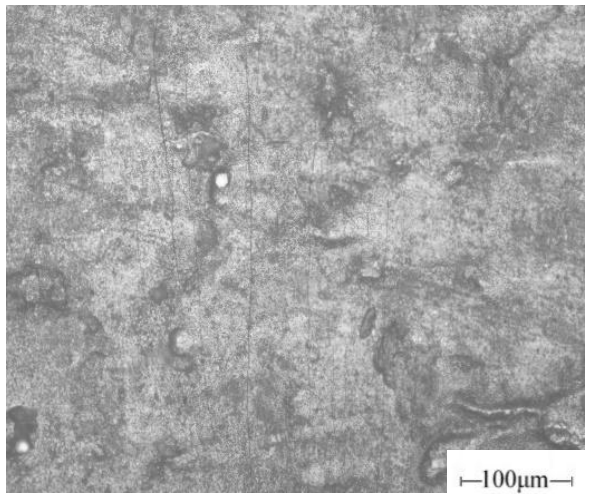

(b)

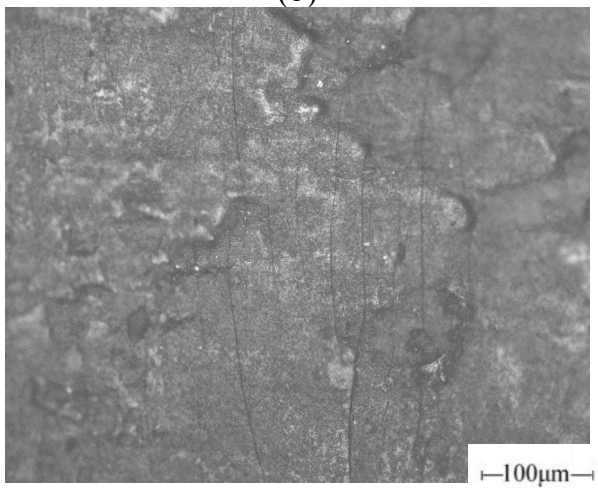

(c)

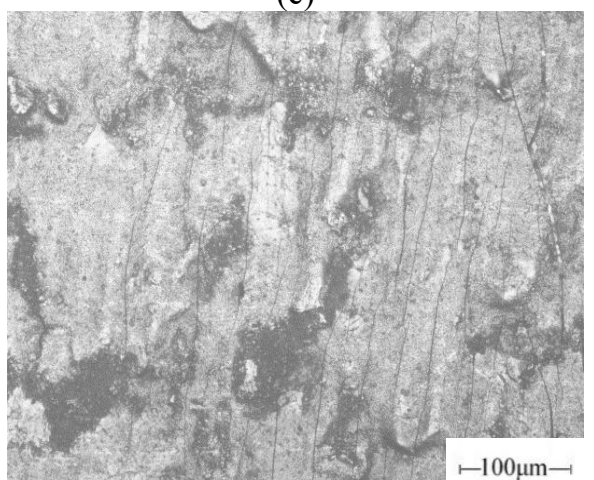

(d)

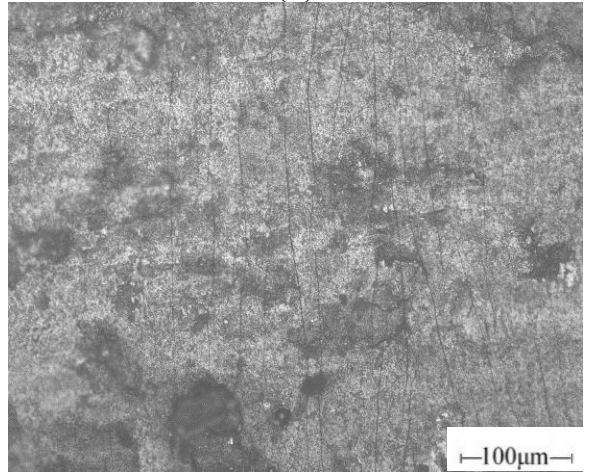

(e) 


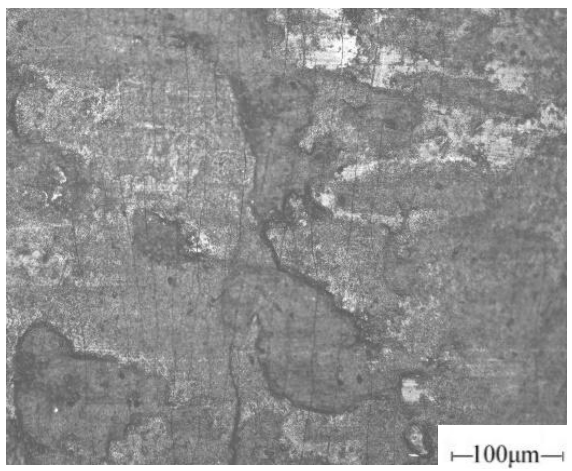

(f)

Fig. 4. Micro-morphologies of oxide scales at different strains: (a)tensile strain $0.05 \%$;(b)tensile strain $0.07 \%$; (c)tensile strain $0.08 \%$;(d)tensile strain $0.10 \%$;(e)tensile strain $0.15 \%$;(f)tensile strain $0.20 \%$.

Fig. 5 is a microscopic topography (10,000 times) of a scale crack observed by a scanning electron microscope. It can be seen from the microscopic topography that the crack width is $2.5 \mu \mathrm{m}$, the lines are neat, and the cracks are scattered with cracking debris.

Fragment of oxide scale

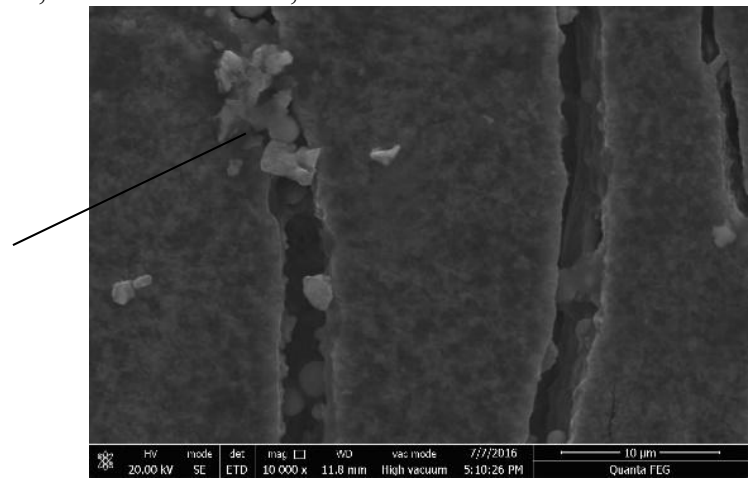

Fig. 5. Crack width in the oxide scale(10,000 times).

\section{4 scale cracking regularity analysis}

The fracture failure behavior of metallic materials under normal temperature tension has two main perspectives.

Fracture mechanics believe that there are micro-defects inside the material and crack growth under external force. When the critical stress $\sigma_{\mathrm{f}}$ reaches the critical condition of formula (3), the material cracks are generated [18]:

$$
\sigma_{\mathrm{f}}=\sqrt{\frac{2 E \gamma}{\pi c}} .
$$

Here, $E$ is the elastic modulus, $\gamma$ is the surface energy, and $c$ is half of the original microscopic crack length of the material.

The study of scale cracking by Evans [8], Robertson and Manning [9], Nagl and Evans [10] was based on the above formula.

The distribution of the oxide layer on the strip surface is relatively uniform and compact, but inevitably there are microscopic defects. The generation of primary cracks and subsequent cracks is caused by the growth of internal defects in the oxide layer under 
external tension. From equation (3), the primary cracks are generated at the maximum defect position, and the smaller defects are followed by cracking with increasing strain. The random defect positions leads to no regularity in the locations of these cracks.

Material mechanics theory are based on the fact that the material is homogeneous and flawless, and it is believed that the fracture failure occurs when the strain $\varepsilon$ of a brittle material reaches its critical value $\varepsilon_{\mathrm{c}}[19]$.

It is assumed that two cracks occur due to the microscopic defects of the oxide layer under tension, the scale layer between the two cracks is free of defects, both the scale layer and the strip steel matrix are continuous and uniform ideal material, and the mechanism and regularity of crack generation in the elastic deformation zone are theoretically deduced.

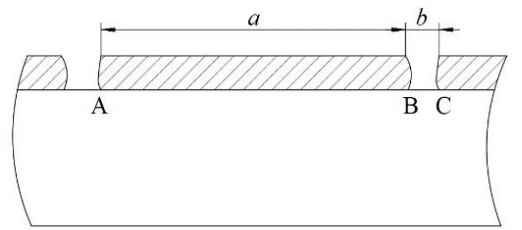

(a)

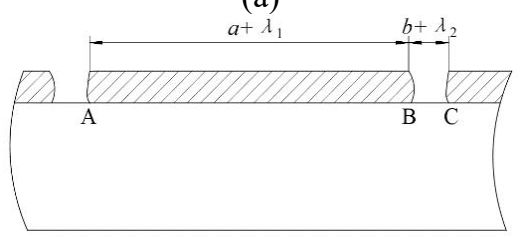

(b)

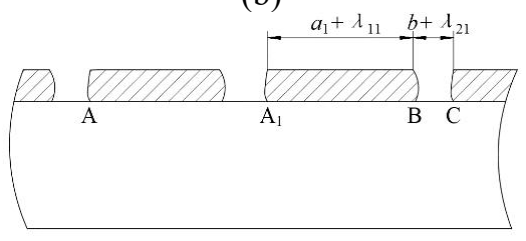

(c)

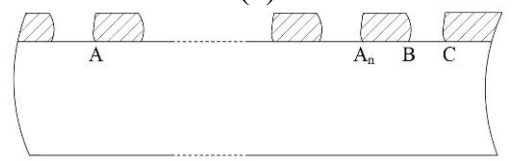

(d)

Fig. 6. Technical principle diagram of oxide scale fracture.

Once the initial cracks are generated, the bonding surface of the scale layer and the strip steel matrix can be divided into two regions. One is the "load zone", and the matrix needs to transfer strain to the oxide layer; and the other is the "no-load zone" at the bottom of the crack. As shown in Fig. 6(a) and 6(b), the "load zone" AB segment (initial width is $a$ ) and the "no-load zone" BC segment (initial width $b$ ) are selected as the study object, when the total deformation is $\lambda$, the $\lambda_{1}$ of the "Load zone" $\mathrm{AB}$ section and the $\lambda_{2}$ of the "no-load zone" $\mathrm{BC}$ segment should be satisfied:

$$
\left\{\begin{array}{l}
\lambda=\lambda_{1}+\lambda_{2} \\
\frac{\lambda_{1}}{a}\left(E_{o}+E_{s}\right)=\frac{\lambda_{2}}{b} E_{s}
\end{array}\right.
$$

In the formula, $E_{\mathrm{o}}$ is the Young's modulus of the scale layer, and $E_{\mathrm{s}}$ is the Young's modulus of the strip steel matrix. At this point, the strain $\varepsilon$ at the AB section of the "load zone" is $\varepsilon=\lambda_{1} / a$. According to equation (4), the following can be obtained: 


$$
\varepsilon=\frac{\lambda}{a+b\left(\frac{E_{\mathrm{o}}}{E_{\mathrm{s}}}+1\right)} .
$$

The strain $\varepsilon$ increases with the total deformation $\lambda$. When the total deformation is increased to $\lambda=\lambda_{0}$, the strain $\varepsilon_{\mathrm{AB}}$ in the "load zone" AB section reaches the critical strain $\varepsilon_{\mathrm{c}}$, that is:

$$
\varepsilon_{\mathrm{AB}}=\frac{\lambda_{0}}{a+b\left(\frac{E_{\mathrm{o}}}{E_{\mathrm{s}}}+1\right)} .
$$

At this point, a new crack will be generated somewhere in the $\mathrm{AB}$ section of the "load zone". Assume that the crack in the natural state is still $b$, and the center of crack is located in the center of the AB section, as shown in Fig. 6(c). The new "load zone" $A_{1} B$ segment deformation $\lambda_{11}$ and the new "no-load zone" BC segment deformation $\lambda_{21}$ at the moment of fracture should be satisfied:

$$
\left\{\begin{array}{l}
\lambda_{11}+\lambda_{21}=\frac{\lambda_{0}}{2} \\
\frac{\lambda_{11}}{a_{1}}\left(E_{\mathrm{o}}+E_{\mathrm{s}}\right)=\frac{\lambda_{21}}{b} E_{\mathrm{s}} .
\end{array}\right.
$$

The $a_{1}$ is the natural state width of the $\mathrm{A}_{1} \mathrm{~B}$ section of the "load zone" and satisfying $a_{1}=(a-b) / 2$.

The new "load zone" $\mathrm{A}_{1} \mathrm{~B}$ segment should be:

$$
\varepsilon^{\prime}=\frac{\lambda_{11}}{a_{1}}
$$

After substituting equation (7), the derivation can be obtained:

$$
\varepsilon^{\prime}=\frac{\lambda_{0}}{a+b\left(2 \frac{E_{\mathrm{o}}}{E_{\mathrm{s}}}+1\right)} .
$$

Comparing with equation (6), it can be seen that $\varepsilon^{\prime}<\varepsilon_{c}$, which indicates that the crack will reduce the strain in the "load zone" and slow down the formation of the next crack.

Defining $\eta$ as the "load zone" deformation distribution rate, with

$$
\eta=\frac{\lambda_{1}}{\lambda_{1}+\lambda_{2}} \text {, and }
$$
substituting it into equation (4), the following can be obtained:

$$
\eta=\frac{1}{1+\frac{b}{a}\left(\frac{E_{\mathrm{o}}}{E_{\mathrm{s}}}+1\right)} .
$$

As shown in Fig. 6(d), after the crack is generated $n$ times, the distribution rate $\eta_{\mathrm{n}}$ of the $\mathrm{A}_{\mathrm{n}} \mathrm{B}$ deformation in the "load zone" can be deduced similarly: 


$$
\eta_{n}=\frac{1}{1+\frac{b}{a_{n}}\left(\frac{E_{\mathrm{o}}}{E_{\mathrm{s}}}+1\right)} .
$$

In this equivalent, $a_{\mathrm{n}}$ is the width of the latest "load zone" $\mathrm{A}_{\mathrm{n}} \mathrm{B}$ segment in its natural state.

Based on the above results, it can be concluded that after the initial cracks are generated, as the stress/strain of the strip increases, the non-defective oxidized iron sheet cracks will continue to be generated. With the gradual generation of cracks, the width " $a_{\mathrm{n}}$ " of the latest "load zone" $\mathrm{A}_{\mathrm{n}} \mathrm{B}$ natural state decreases, resulting in "load zone" deformation distribution rate $\eta_{\mathrm{n}}$ becomes smaller, in other words, the more cracks, the less deformation will be distributed in the "load zone". This will delay the further increase in the number of cracks in the "load zone".

In this experiment, when the strain is less than $0.08 \%$, the number of cracks in the sample increases slowly with the increase of the strain, and there is no regulation in crack position and spacing. These cracks are generated by the growth of defects with different sizes in the oxide layer under tension. When the strain reaches the range of $0.08 \%$ to $0.10 \%$, the cracks increases sharply with increasing strain, which is caused by a large number of non-defective oxidized iron sheet strains reaching the critical strain value and a large amount of cracking occuring along with the increase of strain. When the strain exceeds $0.10 \%$, the number of cracks changes little with strain. This is because the number of cracks increases, so that the "load zone" deformation distribution rate $\eta_{\mathrm{n}}$ becomes smaller, resulting in an increase strain distribution less strain in the "load zone". When the strain exceeds $0.15 \%$, the number of sample cracks is difficult to continue to increase, because the "load zone" deformation rate distribution $\eta_{\mathrm{n}}$ is already very small.

In addition, experiments were conducted to increase the tension of the specimen until the phenomenon of necking occurred. Select a flat surface at a distance of $15 \mathrm{~mm}$ from the constriction to perform wire cutting. Prepare specimens with a length and width of $10 \mathrm{~mm}$. Clean the surface of the specimen with an ultrasonic cleaner, rinse with alcohol, and blow dry with a dryer. The surface of the sample was observed using a ZEISS ULTRA 55 scanning electron microscope to obtain a microscopic topography of the strip shown in Fig.7.

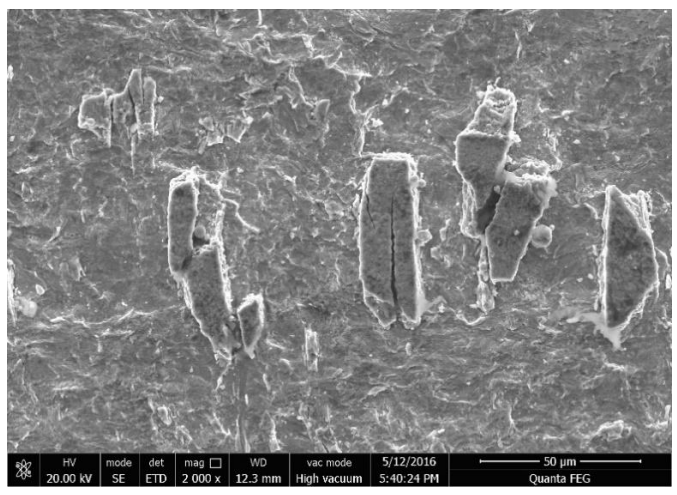

Fig. 7. Microscopic appearance of strip after necking.

In Fig. 7, the strip steel body reaches the yield limit under large tension, and its surface is still firmly attached to a large number of residual scale layers. This observation result is consistent with the above theoretical deduction. With the increase in stress/strain, the scale cracks gradually increase and accompanied with sapling. When a large number of cracks and spalling occur, it can be known from equation (11) that the deformation distribution 
rate $\eta_{\mathrm{n}}$ of the "load zone" is close to zero, in other words, the strip matrix deformation should not be transmitted to the scales, which creates a self-locking phenomenon. Therefore, it is unrealistic to rely solely on tension to achieve higher cleanliness of descaling, which is consistent with the statistical conclusions of Li and Wang [6-7].

Abrasive water jet descaling is a typical acid-free descaling technique that relies on the kinetic energy of the abrasive stream to impact and remove the brittle oxide layer on the substrate surface. A large number of cracks appear on the surface of the strip steel under tension, which facilitates the cleaning of the abrasive flow. However, if the strain is too big, the crack density will not be significantly increased. Instead, the energy consumption of the system will increase. At the same time, the scale layer will peel off from the substrate. This will inevitably cause the abrasive flow to directly impact the substrate and cause unnecessary metal consumption. In summary, in the pretreatment process of the abrasive water jet, a reasonable range of tension setting should be $0.15 \%-0.18 \%$.

\section{Conclusion}

(1)The scale layer has no obvious delamination, and the structure is dense and wellintegrated with the substrate. The thickness is about $10 \mu \mathrm{m}$. The direction of the crack generated under tension is approximately perpendicular to the tension direction. After the stress is released, the crack width is about $2.5 \mu \mathrm{m}$. The lines are neat. Cracks scattered with cracked chips.

(2)The initial cracking of the specimen under tension is caused by the growth of the inner oxide layer. With the increase of the strain, the oxide layer strain at the non-defected area reaches the critical strain and cracking occurs. With increasing strain, the number of scale cracks on the surface of the sample increases at first slowly, then fast, and finally slowly. The strain corresponding to the primary crack is $0.05 \%$. When the strain is bigger than $0.15 \%$, the number of cracks increases very slowly. This is due to cracks. The increase in the number of strips leads to a significant reduction in the distribution rate $\eta_{\mathrm{n}}$ of the "load zone" deformation.

(3)In the pretreatment process of non-pickling and descaling, the excessive strain should not increase the crack density, but will increase the energy consumption of the system. At the same time, it will lead to the partial scale layer peeling off the matrix, which will inevitably cause the direct impact of the abrasive flow. Strip surface, causing unnecessary metal consumption.

\section{Acknowledgments}

This work was supported by Beijing Natural Science Foundation(3182026), National Natural Science Foundation of China (Grant No.51604024), The Tribology Science Fund of State Key Laboratory of Tribology (Grant No.SKLTKF16B11), Fundamental Research Funds for the Central Universities (Grant No.FRF-TP-17-002A2).

\section{References}

1. Gleeson B, Hadavi S M M, Young D J. Isothermal transformation behavior of thermally-grown wüstite[J]. Materials at High Temperatures, (2000), 17(2): 311

2. Schwerdtfeger K, Shunxin Z. A contribution to scale growth during hot rolling of steel[J]. Steel research international, (2003), 74(9): 538

3. Lin G N, Chen X Y, Tang B, et al. The cleaner production of pickling Steel for iron 
steel parts with rust in fix quantify[J]. Guangdong Chemical Industry, (2008), 35(5): 56-60.

4. Wang Y J, Dong H J, Mu H L, et al. Analysis on scale microstructure of coiled hot rolled strip. China Metall, (2007),17(10): 40

5. Melster S, Pargmann A. Stretch bending levelers in pickling lines[J]. Iron Steel Eng., (1995), 72(2): 37-41.

6. Wang J G, Li T Q. Mechanical analysis of stretching and straightening deformation processes of strip steel. Shanghai Metal, (1998), 20(5): 35

7. Li T Q. Study on stretch-bend levelling theory and technology of the pickling tension leveler. Metall Equip, (2011)(1): 19

8. Evans H E. Cracking and spalling of protective oxide layers[J]. Materials Science and Engineering: A, (1989), 120: 139

9. Robertson J, Manning M I. Limits to adherence of oxide scales[J]. Materials Science and Technology, (1990), 6(1): 81

10. Nagl M M, Evans W T. The mechanical failure of oxide scales under tensile or compressive load[J]. Journal of Materials Science, (1993), 28(23): 6247

11. Chaudhuri S K, Rolls R. Fracture mechanisms in oxide scale on iron during substrate deformation. J Mater Sci, (1977), 12(11): 2303

12. Zhang Q D, Huang L W, Wu B, et al. Experimental research on the surface scale of hot rolled steel strip. Shanghai Metal, (2000), 22(5): 32

13. Bian D P, Zhou C L, Li Z X, et al. Study on theory of descaling by tension-bending leveler. Mech Eng Autom, (2013)(6): 86

14. He Y Q, Jia T, Liu Z Y, et al. Fracture behavior of surface oxide scale in cold rolling. J Northeastern Univ ( Nat Sci), (2013), 34(7): 948

15. Le H R, Sutcliffe M P F, Wang P Z, et al. Surface oxide fracture in cold aluminium rolling. Acta Mater, (2004), 52(4): 911

16. Chen R Y, Yeun W Y D. Review of the high-temperature oxidation of iron and carbon steels in air or oxygen. Oxid Metal, (2003), 59(5-6): 433

17. Gu Q D, Dong C F, Li X G, et al. Corrosion behavior and structure of oxide scales formed on hot rolled strips after coiling with different oxygen supplies. J Univ Sci Technol Beijing, (2009), 31(12): 1564)

18. Kanninen M F, Popelar C H. Advanced Fracture Mechanics. New York: Oxford University Press, Oxford: Clarendon Press, (1985)

19. Liu H W. Mechanics of Materials. 4th Ed. Beijing: Higher Education Press, (2004) 